\section{Bayes, Satz von}

R.-D. Hilgers ${ }^{1}$, N. Heussen ${ }^{1}$ und S. Stanzel ${ }^{2}$

${ }^{1}$ Institut für Medizinische Statistik, Universitätsklinikum der RWTH Aachen, Aachen, Deutschland

${ }^{2}$ DKFZ Heidelberg, Heidelberg, Deutschland

Synonym(e) Bayes-Theorem

\section{Englischer Begriff Bayes theorem}

Beschreibung Im 18. Jahrhundert interessierte sich der Geistliche und Mathematiker Thomas Bayes für die Wahrscheinlichkeit, dass ein Ereignis eintritt, wenn eine bestimmte Bedingung vorliegt. Diese Wahrscheinlichkeit wird heutzutage als bedingte Wahrscheinlichkeit bezeichnet. Ein Ergebnis seiner Forschung besteht in der Formulierung des sog. Bayes-Theorems (Satz von Bayes). Dieses beschreibt, wie man zu einer neuen Bewertung gelangt, wenn man die Beurteilung der Ereignisse eines Experimentes vor Durchführung (A-priori-Annahme) mit den beobachteten Ergebnissen kom- biniert. $\mathrm{Zu}$ den Standardbeispielen des Bayes-Theorems gehören die Probleme der „probabilistischen Kategorisierung". Dabei handelt es sich um die Aufgabe der Klassifizierung unter Unsicherheit. Ein solches Problem liegt in typischer Weise im Rahmen diagnostischer Tests vor. Hier beschreibt der Satz von Bayes den Zusammenhang zwischen der Diagnose bevor der Test durchgeführt wurde und der Diagnose bei Vorliegen des Ergebnisses eines diagnostischen Tests.

Der Satz von Bayes wird häufig verwendet, um den $\mathrm{Zu}-$ sammenhang zwischen prädikativen Werten, der Sensitivität ( $\triangleright$ Sensitivität, diagnostische), der Spezifität ( $\triangleright$ Spezifität, diagnostische) und der $\triangleright$ Prävalenz zu beschreiben $(\triangleright$ Vorhersagewert, positiver; $\triangleright$ Vorhersagewert, negativer).

\section{Literatur}

Bayes TR (1958) An essay towards soving a problem in the doctrine of chance. (Original 1763). Biometrika 45:293-315

Hilgers R-D, Bauer P, Scheiber V (2002) Einführung in die Medizinische Statistik. Springer, Berlin/Heidelberg/New York 